\title{
Sur le paramagnétisme de l'ion de cobalt en solutions très diluées
}

\section{Doctoral Thesis}

Author(s):

Mercier, Robert

Publication date:

1934

Permanent link:

https://doi.org/10.3929/ethz-a-000091731

Rights / license:

In Copyright - Non-Commercial Use Permitted 


\title{
S UR
}

\section{LE PARAMagNÉTISME DE L'ION DE COBALT} EN SOLUTIONS TRÈS DILUÉES

\section{THĖSE}

PRESENIÉE A L'ÉCOLE POLYTECHNIQUE FÉDÉRALF, ZURICH POUR L'OBTENTION DU

GRADE DE DOGTEUR ĖS SCIENGES NATURELLES PA R

\section{R. MERCIER}

Ing. dipl. E. I. L. de Genère.

\author{
Rapporteur : Prof. Dr P. SCHERIREIR. \\ Co-Rapporteur : Prof. Dr F. TANK.
}

\section{PARIS}

MASSON ET C ${ }^{\text {ie }}$, ÉDITEURS

LIBRAIRES DE L'ACADÉMIE DE MÉDEGINE 120, BOULEVARD SAINT-GERMAIN 
un demi-magnéton de Weiss ( $\left.{ }^{1}\right)$. La précision est d'aviant meilleure que la température est moins basse.

\$ 7. Conclusions. - En résumé les points suivants sont acquis :

a) En solutions dans le méthanol, le $\mathrm{CoCl}_{2}$ présente, pour des titres variant entre $0,002760 \mathrm{~g} \cdot / \mathrm{g}$. et $0,000138 \mathrm{~g} / \mathrm{g}$. un coefficient d'aimantation spécifique pratiquement indépendant de la concentration.

b) Le moment magnétique, calculé selon Van Vleck, varie avec la température.

c) Il semble que près du point de fusion seul le spin soit magnétiquement actif $\left({ }^{2}\right)$.

Le fait que la théorie du magnétisme des cristaux semble pouvoir s'appliquer à une solution n'a rien qui doive surprendre. En effel c'est l'ordination spéciale des atomes immédiatement voisins qui donne au champ électrique perturbateur son caractère de symétrie. Or on a trouvé ( $\left.{ }^{3}\right)$ par l'étude aux rayons $X$ que cette ordination spatiale existait en moyenne autour de chaque particule liquide. Il est probable que la formation de complexes de Co avec un indice de coordination 6 n'est que le résultat de l'exaltation de cette propriété.

Au surplus, Gorter (*) a montré que le groupement de 6 dipóles électriques placés perpendiculairement aux 6 faces du cube, donnait naissance au centre de celui-ci à un champ qui selon Bethe est justement capable en principe de " geler " le moment orbital de l'ion qui s'y trouve.

Si l'énergie de couplage de ces édifices supermoléculaires est de l'ordre de grandeur de $k \mathrm{~T}$, on peut prévoir que les

(1) Soit o, I magnéton de Bohr.

(2) Voir p. 26.

(3) Amaldi, Phys. Z $z c h .\left(9^{31}\right)$, p. 914 ; Debye et H. Menke, Phys. Zsch. (ig3o), p. 797 .

(') Gorter, Phys. Rev., 42, ( $\left.\mathrm{rg}^{32}\right)$, p. 437 . 
chocs moléculaires les détruiront facilement et leur durée moyenne sera fonction de la température. Il s'ensuit que pour un seul et même sel, le moment magnétique sera variable non seulement avec la température mais aussi avec la nature du liquide dans lequel il sera dissous. Autrement dit, les phénomènes sont trop complexes chez les sels en solution pour pouvoir d'une seule étude thermomagnétique, conclure de l'élat électronique du ion. Une étude détaillée de l'unicristal pourra au contraire être très féconde.

Je ne voudrais pas conclure sans exprimer toute ma gratitude à mon maitre, M. le professeur Scherrer sous la dírection de qui ce travail a été exécuté, pour tout l'intérêt et les précieux conseils qu'il m'a prodigués.

Zurich, Institut de Physique de l'Ecole Polytechnique Féderale, juillet rg.33. 